\title{
HAMILTONIAN CYCLES IN STRONG PRODUCTS OF GRAPHS
}

\author{
BY \\ J. C. BERMOND, A. GERMA, AND M. C. HEYDEMANN
}

\begin{abstract}
Let $\overline{\times} G^{k}$ denote the graph $G \overline{\times} G \overline{\times} \cdots \overline{\times} G$ ( $k$ times) where $G \overline{\times} H$ is the strong product of the two graphs $G$ and $H$. In this paper we prove the conjecture of J. Zaks [3]: For every connected graph $G$ with at least two vertices there exists an integer $k=k(G)$ for which the graph $\overline{\times} G^{k}$ is hamiltonian.
\end{abstract}

Let $G$ be a graph (undirected) and let $V(G)$ and $E(G)$ denote the vertex set and the edge set of $G$. The strong product $G \overline{\times} H$ of two graphs $G$ and $H$ is defined by

$$
V(G \overline{\times} H)=V(G) \times V(H)
$$

$E(G \overline{\times} H)=\left\{\left\{\left(u_{1}, v_{1}\right)\left(u_{2}, v_{2}\right)\right\} \mid u_{1}, u_{2} \in V(G), v_{1}, v_{2} \in V(H)\right.$ and

either $u_{1}=u_{2}$ and $\left\{v_{1}, v_{2}\right\} \in E(H)$

either $v_{1}=v_{2}$ and $\left\{u_{1}, u_{2}\right\} \in E(G)$

either $\left\{u_{1}, u_{2}\right\} \in E(G)$ and $\left.\left\{v_{1}, v_{2}\right\} \in E(H)\right\}$.

This product is commutative and associative and following [3] we shall denote by $\overline{\times} G^{k}$ the graph $G \overline{\times} G \overline{\times} \cdots \overline{\times} G$ ( $k$ times).

In [3] J. Zaks proved that: "For every $h$ and $k, h \geq 1, k \geq 1$, there exists an $h$-connected graph $G=G(h, k)$, such that the graph $\overline{\times} G^{k}$ is non-hamiltonian" and asked:

"Is it true that for every connected graph $G$ with at least two vertices there exists an integer $k=k(G)$ for which the graph $\overline{\times} G^{k}$ is hamiltonian".

We give an affirmative answer to this question in Theorem 11, the proof of which needs Lemmata and Propositions 1 to 10.

Our notations are as follows:

$-P_{n}$ the path with $n$ vertices.

$-d_{G}(x)$ the degree of the vertex $x$ in $G$.

$-\Delta(G)$ the maximum degree of the vertices of $G$.

- For $a$ in $V(H)$ (resp. $b$ in $V(G)) G_{a}$ (resp. $H_{b}$ ) denote the subgraph $G \overline{\times}\{a\}$ of $G \overline{\times} H$ (resp. $\{b\} \overline{\times} H$ of $G \overline{\times} H)$. 
The reader is referred to C. Berge [1] for any graph theory terms not defined here.

LEMMA 1. The strong product of two connected graphs is connected.

Lemma 2. For every graph $G$ and every integer $n \geq 2$, there exists a covering of the vertices of $G \overline{\times} P_{n}$ by vertex-disjoint subgraphs isomorphic to $P_{n}$.

Proof. The subgraphs of the covering are $\left(P_{n}\right)_{a}$ with $a \in V(G)$.

LEMMA 3. For every $n$ and $m, 2 \leq n<m$, there exists a covering of the vertices of $K_{1, n} \overline{\times} K_{1, m}$ by vertex-disjoint subgraphs isomorphic to $K_{1, n}$.

Proof. The subgraphs of the covering are $\left(K_{1, n}\right)_{a}$ with $a \in V\left(K_{1, m}\right)$.

LEMma 4. For every $n, n \geq 3$, there exists a covering of the vertices of $\overline{\times} K_{1, n}^{2}$ by vertex-disjoint subgraphs isomorphic to $K_{1, n_{\mathrm{i}}}$ with $n_{i}<n$.

Proof. The general construction is an easy generalization of decomposition shown in Fig. 1 for $n=5$.

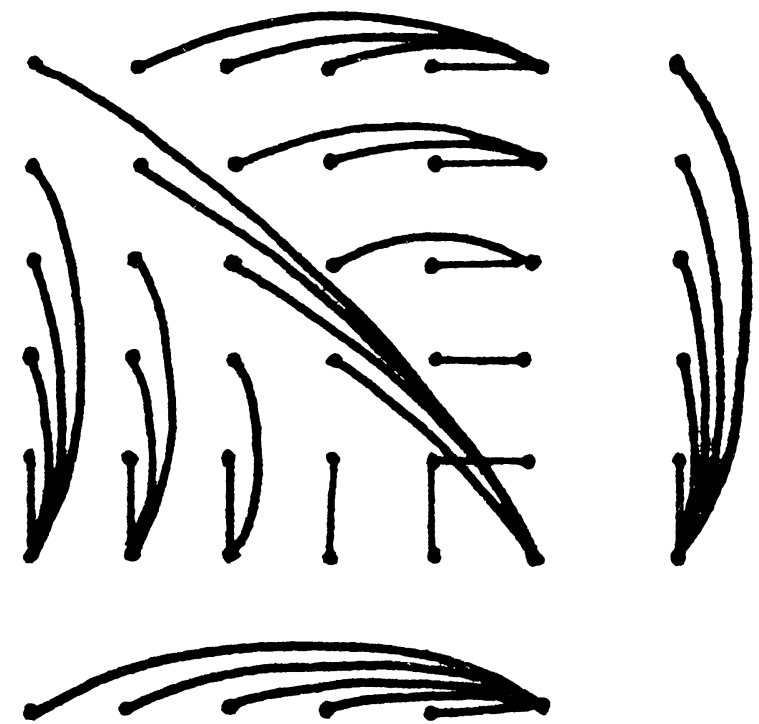

Figure 1

Proposition 5. For every connected graph $G$, there exists an integer $k_{1}=k_{1}(G)$ such that $V\left(\overline{\times} G^{k_{1}}\right)$ can be covered by vertex-disjoint paths of positive length.

Proof. Every non empty graph $G$ with no isolated vertices is vertex covered by disjoint paths of positive length and by stars, as can be easily shown by induction on the number of vertices of $G$ (or by considering a maximal matching of $G$ ). Let the disjoint paths $P_{n_{i}}, i \in I$, of positive length and stars 
$K_{1, n}, j \in J$, cover all vertices of $G$. If $J=\varnothing$ the proposition is true for $k_{1}=1$. If $J \neq \varnothing, V\left(\overline{\times} G^{2}\right)$ can be covered by vertex-disjoint subgraphs of the following types: $P_{n_{i}} \overline{\times} P_{n_{i}}, P_{n_{i}} \overline{\times} K_{1, n_{i}}, K_{1, n_{i}} \overline{\times} K_{1, n_{i}}$.

Then, as a consequence of Lemmata 2, 3, 4, $V\left(\overline{\times} G^{2}\right)$ admits a covering by vertex-disjoint subgraphs isomorphic to paths of positive length and stars $K_{1, n_{1}}$, $l \in L$ with

$$
\max \left\{n_{l}, l \in L\right\} \leq \max \left\{n_{j}, j \in J\right\}-1 .
$$

Since $\overline{\times} G^{2}$ is connected (Lemma 1) an easy induction on $\max \left\{n_{\mathrm{j}}, j \in J\right\}$ shows that an integer $k_{1}$ exists, as required; in fact, $k_{1}$ can be chosen to satisfy $k_{1} \leq 2^{\Delta(G)-2}$.

LEMMA 6. For every $n$ and $m, n, m \geq 2, P_{n} \overline{\times} P_{m}$ admits a hamiltonian cycle (of length $\mathrm{nm}$ ).

Proof. The construction of a hamiltonian cycle in $P_{n} \overline{\times} P_{m}$ is an immediate generalization of one of the two following constructions of Fig. 2.

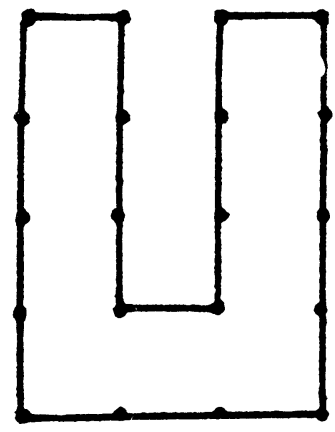

$\mathrm{n}$ or $\mathrm{m}$ eren

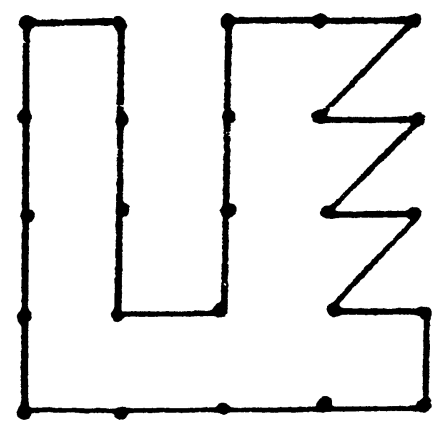

$\mathrm{n}$ and $\mathrm{m}$ odd

Figure 2

Proposition 7. Let $G$ be a graph of maximum degree $\Delta(G)$; if there exists a covering of $V(G)$ by vertex-disjoint paths of positive length, then there exists an integer $k_{2}=k_{2}(G)$ such that $V\left(\overline{\times} G^{k_{2}}\right)$ can be covered by vertex-disjoint cycles of length at least $\Delta(G)$.

Proof. Let us consider a covering of $V(G)$ by vertex-disjoint paths $P_{n_{i}}, i \in I$, $n_{i} \geq 2$. Then, by Lemma $6, V\left(\overline{\times} G^{2}\right)$ admits a covering by vertex-disjoint cycles of length at least $\left(\operatorname{Inf}_{i \in I} n_{i}\right)^{2}$ and thus by paths with at least $\left(\operatorname{Inf}_{i \in I} n_{i}\right)^{2}$ vertices. Then, by induction, $\overline{\times} G^{p}$ can be covered by vertex-disjoint cycles of length at least $\left(\operatorname{Inf} n_{i}\right)^{p}$. As $\operatorname{Inf}_{i \in I} n_{i} \geq 2$, there exists an integer $k_{2}$ such that $\left(\operatorname{Inf}_{i \in I} n_{i}\right)^{k_{2}} \geq$ $\Delta(G)$

As pointed out by the referee the following lemma is similar to lemma 5 of [2] (see also the proof of theorem 4 in [2]). 
LEMMA 8. Let $T$ be a tree of maximum degree $\Delta(T)$ and $C$ be a cycle of length $k \geq \Delta(T)$, then $T \overline{\times} C$ is hamiltonian and there exists a hamiltonian cycle which uses, for any vertex $u \in V(T)$ exactly $k-d_{T}(u)$ edges of $C_{u}$.

Proof. By induction on $|V(T)|$.

When $T=\{u\},\{u\} \overline{\times} C$ is isomorphic to $C$, so the result is true.

If $|V(T)|>1$, let $u$ be an end vertex of $T$ and $v$ its neighbour in $T$, let $T^{\prime}$ be the subtree induced by $V(T)-\{u\}$. By induction hypothesis, $T^{\prime} \overline{\times} C$ admits a hamiltonian cycle which uses $k-d_{T^{\prime}}(v)=k-\left(d_{T}(v)-1\right)$ edges of $C_{v}$. Since $k \geq \Delta(T), k-d_{T^{\prime}}(v) \geq 1$, so there exists an edge $\left\{\left(v, c_{1}\right)\left(v, c_{2}\right)\right\}$ of this hamiltonian cycle, with $\left\{c_{1}, c_{2}\right\}$ in $E(C)$.

Then we construct a hamiltonian cycle in $T \overline{\times} C$ by replacing this edge by the following path:

- the edge $\left\{\left(v, c_{1}\right)\left(u, c_{1}\right)\right\}$

- the path obtained by removing the edge $\left\{\left(u, c_{1}\right)\left(u, c_{2}\right)\right\}$ of the cycle $C_{u}$

- the edge $\left\{\left(u, c_{2}\right)\left(v, c_{2}\right)\right\}$.

This cycle uses for any vertex $u$ in $T$ exactly $k-d_{T}(u)$ edges of $C_{u}$.

REMARK. Lemma 8 gives an iterative method to construct a hamiltonian cycle of $T \overline{\times} C$. Furthermore, by starting the construction with $u$ and $v$ we have that, for any edge $\{u, v\}$ of $T$ and for any edge $\left\{c_{1}, c_{2}\right\}$ of $C$, there exists a hamiltonian cycle in $T \overline{\times} C$ which uses the two following edges $\left\{\left(u, c_{1}\right)\left(v, c_{1}\right)\right\}$ and $\left\{\left(u, c_{2}\right)\left(v, c_{2}\right)\right\}$.

Corollary 9. If $\overline{\times} G^{k}$ is hamiltonian, then for every $p \geq 0, \overline{\times} G^{k+p}$ is hamiltonian too.

Proof. By induction on $p$. Lemma 8 applied with $T$ a spanning tree of $G$ and $C$ a hamiltonian cycle in $\overline{\times} G^{k}$ shows that $\overline{\times} G^{k+1}$ is hamiltonian.

Corollary 9 is true even for just the cartesian product and it has been proved in [3] as theorem 1.

Proposition 10. If $G$ is connected and if there exists a covering of $V(G)$ by $\alpha$ vertex-disjoint cycles of length at least $l$, with $\alpha \geq 2$, then for every tree $T$ with at least two vertices and with $\Delta(T) \leq l$, there exists a covering of $V(G \overline{\times} T)$ by $\beta$ vertex-disjoint cycles of length at least $l$ with $\beta \leq \alpha-1$.

Proof. Since $G$ is connected, there exists two cycles of the covering $C_{1}$ and $C_{2}$, and vertices $c_{1}$ in $V\left(C_{1}\right)$ and $c_{2}$ in $V\left(C_{2}\right)$ with $\left\{c_{1}, c_{2}\right\}$ in $E(G)$.

By Lemma 8 , there exists a covering of $V(G \overline{\times} T)$ by $\alpha$ hamiltonian cycles of $C_{i} \overline{\times} T$. To prove the proposition it suffices to construct a hamiltonian cycle in $V\left(C_{1} \overline{\times} T\right) \cup V\left(C_{2} \overline{\times} T\right)$.

Let $\{u, v\}$ be an edge of $T$. By the remark of Lemma 8 , we can construct a 
hamiltonian cycle $H_{1}$ in $C_{1} \overline{\times} T$ (resp. $H_{2}$ in $C_{2} \overline{\times} T$ ) which uses the edge $\left\{\left(u, c_{1}\right)\left(v, c_{1}\right)\right\}$ (resp. $\left.\left\{\left(u, c_{2}\right)\left(v, c_{2}\right)\right\}\right)$. We obtain a cycle in $V\left(C_{1} \overline{\times} T\right) \cup V\left(C_{2} \bar{x} T\right)$ by replacing in $E\left(H_{1}\right) \cup E\left(H_{2}\right)$ the preceding edges by $\left\{\left(u, c_{1}\right)\left(u, c_{2}\right)\right\}$ and $\left\{\left(v, c_{1}\right)\left(v, c_{2}\right)\right\}$.

THEOREM 11. For any connected graph $G$ with at least two vertices there exists an integer $k=k(G)$ for which the graph $\overline{\times} G^{k}$ is hamiltonian.

Proof. By Proposition 5, there exists an integer $k_{1}$ such that there exists a covering of $V\left(\overline{\times} G^{k_{1}}\right)$ by vertex-disjoint paths, with at least two vertices; then by Proposition 7 applied to $\bar{x} G^{k_{1}}$, there exists an integer $k_{2}$ such that $V\left(\bar{x} G^{k_{1} k_{2}}\right)$ can be covered by $\alpha$ vertex-disjoint cycles of length at least $\Delta(G)$ (since $\Delta\left(G^{k_{1}}\right) \geq \Delta(G)$ ).

Repeated applications of Proposition 10 show that there exists an integer $k_{3} \leq \alpha-1$ such that $\bar{x} G^{k_{1} k_{2} k_{3}}$ is hamiltonian. Thus Theorem 11 is proved with $k=k_{1} k_{2} k_{3}$.

REMARK. The integer $k$ found in the proof of theorem 11 is not the best possible. We conjecture that:

CONJECTURE. For any connected graph $G$ with at least two vertices $\overline{\times} G^{\Delta(G)}$ is hamiltonian.

AcKnowledgement. We thank Professor J. Zaks for having drawn our attention to the article [2].

\section{REFERENCES}

1. C. Berge. Graphs and Hypergraphs, North-Holland. Amsterdam 1973.

2. M. Rosenfeld and D. Barnette, Hamiltonian Circuits in Certain Prisms, Discrete Math. 5, 1973, 389-394.

3. J. Zaks. Hamiltonian cycles in products of graphs, Canadian Math. Bull. vol. 17 (5), 1975, 763-765.

UNIVERSITÉ PARIS-SUD

INFORMATIQUE, BÂTIMENT 490

91405. Orsay, France 